\title{
Trender i antal ringmärkta fåglar av fyrtio arter på Stora Fjäderäggs fågelstation, nordöstra Sverige, 1985-2014
}

\author{
Trends in ringing numbers of forty bird species at Stora Fjäderägg Bird \\ Observatory, north-eastern Sweden, 1985-2014
}

\author{
LARS EDENIUS, NIKLAS LINDBERG ALSERYD \& SÖREN WULFF
}

\begin{abstract}
Very few data series are available on the long-term development of the bird fauna in northern Sweden. This kind of data is of great interest as there are recent signs that bird populations in northern Sweden are developing less favourable than in southern Sweden. We present trends in annual capture rates of 40 frequently ringed species at Stora Fjäderägg Bird Observatory, NE Sweden, autumns 1985-2014. Significant positive and negative trends were found in ten and eight species, respectively. Great tit, Chaffinch and Long-tailed Tit showed the strongest increases, whereas Northern Wheatear, Willow Tit and Bluethroat decreased the most. There was a significant negative trend in capture index for short-distance migrants and a positive trend for irruptive species/partial

migrants. For many species, particularly those that were decreasing, the trends at Stora Fjäderägg are similar to population trends seen in Swedish and Finnish breeding bird surveys. For these species the trends at Stora Fjäderägg could be indicative of long-term population changes.

Lars Edenius, Snickargatan 12, 90360 Umeå, lars.edenius@grubbe.com Niklas Lindberg Alseryd, Orgelstråket 13, 90353 Umeå, nlindbergalseryd@gmail.com Sören Wulff, Kopparvägen 42T, 90750 Umeå, wulfssoren@gmail.com
\end{abstract}

Received 11 March 2015, Accepted 24 June 2015, Editor: J. Waldenström

\section{Inledning}

Långa dataserier som beskriver utvecklingen av fågelpopulationer över tiden är viktiga för att förstå den inneboende dynamiken i fågelsamhällen, identifiera ekologiska mönster, kunskapsbrister och eventuella hotbilder. Från Ammarnäsområdet, Lycksele lappmark, norra Sverige, finns unikt långa dataserier på häckfåglar i fjällbjörkskog och fjällhed (t.ex. Enemar m.fl. 2004, Svensson \& Andersson 2013). Från resten av Norrland finns mycket få långa dataserier på fåglar redovisade. Svensk Fågeltaxering (SFT) kommer med tiden att svara upp mot detta behov, men standardrutterna - den mest tillförlitliga datakällan - har inte varit i funktionell drift längre än dryga tio år i norra Sverige (Green \& Lindström 2015a). Dessutom är antalet fria punktrutter, en datakälla som sträcker sig längre tillbaka i tiden, få i den norra delen av landet (Ottvall m.fl. 2009). Trendanalyser baserade på långa tidsserier är särskilt viktiga i norra Sverige där fågelpopulationer verkar utvecklas mer negativt än i södra Sverige (Green \& Lindström 2015a, b). Dessutom är utvecklingen hos fågelpopulationer i norr generellt mindre känd jämfört med i de västra och centrala delarna av Europa (Vickery m.fl. 2014).

Ringmärkningsdata har använts för analys av populationsförändringar och för att öka vår förståelse av viktiga skeenden i fågelpopulationerna (t.ex. Busse 1990, Berthold \& Fiedler 2005). För södra Sverige har trendanalyser baserade på ringmärkningsdata presenterats från Falsterbo och Ottenby fågelstationer (1980-1999; Karlsson m fl. 2002a,b, Stervander m.fl. 2005, Jonzén m.fl. 2006). Fåglar som fångas under sträcktid på fågelstationerna kommer ifrån ett delvis okänt rekryteringsområde. Analys av återfynd av fåglar ringmärkta på Stora Fjäderäggs fågelstation i nordöstra Sverige antyder att våra nordliga och sydliga fågelstationer berörs av olika flyttfågelflöden (Elmberg m.fl. 2005) och betonar behovet av regionaliserade analyser av ringmärkningsdata. Det måste dock beaktas att ringmärkningsdata inte alltid är pålitliga indikatorer på förändringar i populationsstorlek (Dunn m.fl. 1997, Hochachka \& Fiedler 2008).

$\mathrm{Vi}$ redovisar här fångsttrender baserade på 30-åriga ringmärkningsdata från Stora Fjäderäggs 
fågelstation, nordöstra Sverige. Fyrtio mer frekvent förekommande arter ingår $i$ analyserna, däribland arter som nästan inte alls fångas på fågelstationerna i södra Sverige som tallbit Pinicola enucleator och videsparv Emberiza rustica. Trenderna för aktuella arter 1985-2014 jämförs med populationstrender i hela Sverige (SFT perioden 1976-2007; Ottvall m.fl. 2009). SFT 1976-2007 är en semi-kvantitativ sammanvägning av olika datakällor, främst häckfågeldata baserade på punkt- och standardruttsdata, men även data från fågelstationer och annat håll ingår. Vi kompletterar uppgifterna i SFT 1976-2007 med trender beräknade för Sverige 1998-2014 (SFT 1998-2014, Green \& Lindström 2015a) och SFT Norrbotten 1988-2013 (Green \& Lindström 2015b), vilka helt baseras på standardruttsdata, för att få bättre tidsmässig och geografisk täckning i jämförelserna. Vi har ingen entydig bild av varifrån fåglarna som fångas på Stora Fjäderägg kommer; kontroller av fåglar ringmärkta tidigare samma höst indikerar dock ett betydande inflöde från ost och sydost (t.ex. kungsfågel Regulus regulus, talgoxe Parus major, blåmes Cyanistis caeruleus och stjärtmes Aegithalos caudatus) - alltså utanför Sveriges gränser för vissa arter. Vi inkluderar därför även populationsdata från finska häckfågeltaxeringar (FHT, 1986-2012, Laaksonen \& Lehikoinen 2013) i våra analyser. Utöver enskilda arter redovisar vi även analys av trender för arterna grupperade efter flyttningsstrategi eftersom denna aspekt av fåglarnas ekologi verkar ha betydelse för populationsutvecklingen (Laaksonen \& Lehikoinen 2013, Vickery m.fl. 2014).

\section{Material och metoder}

\section{Ringmärkningen på Stora Fjäderäggs fågelstation}

Stora Fjäderägg är en ca. $1 \times 1 \mathrm{~km}$ stor vegetationsfattig ö belägen i Norra Kvarken nordost om Holmöarna, Västerbottens län $\left(63^{\circ} 49^{\prime} \mathrm{N}, 21^{\circ} 00^{\prime} \mathrm{E}\right)$. Här i den Bottniska vikens smalaste del fungerar Holmöarkipelagen och Österbottens vidsträckta skärgård som en sträckledare mellan Sverige och Finland. Detta manifesteras i nationellt mycket höga summor av kungsfågelsångare Phylloscopus proregulus, tajgasångare Phylloscopus inornatus, sibirisk piplärka Anthus hodgsoni m.fl. östliga vagranter i höstfångsten på Stora Fjäderäggs fågelstation.

Stora Fjäderägg etablerades som fågelstation 1984, men den mer regelbundna ringmärkningsverksamheten kom igång först ett år senare. Aktivi- teten var hög både vår och höst under de inledande åren men därefter har höstmärkningen helt dominerat. Fångst för ringmärkning sker uteslutande med slöjnät (12 meter långa). Nätplatser och antal nät har varierat över åren men har legat på runt tio respektive 25-30. Nätfångst bedrivs normalt från gryningen fram till lunchtid. Åren 2004-2008 prövades en fälla av helgolandstyp, men den var tämligen ineffektiv ur fångstsynpunkt och krävde en hel del underhåll varför den avvecklades. Under åren fällan var i drift var ett par nätplatser avvecklade och utnyttjandet av fällan gav inte några uppenbara effekter på fångsten (Figur 1).

Ringmärkningsverksamheten på fågelstationen sker helt ideellt och bemanningen har därför varierat över åren. Antalet bemanningsdagar under augusti, september och oktober 1985-2014 låg på i medeltal 78 dagar per år, utan någon långsiktig trend ( $p>0.05$, linjär regression). Antalsmässigt dominerar kungsfågel, gråsiska Carduelis flammea och lövsångare Phylloscopus trochilus (acredula) i fångsten, men även "norrlandsspecialiteter" som videsparv och tallbit ringmärks i nationellt höga antal.

\section{Urval av arter och trendberäkningar}

Vi analyserade trender i höstmärksummorna av de 40 mest frekvent fångade arterna på Stora Fjäderäggs fågelstation höstarna 1985-2014. Här ingår dock inte de två vanligaste arterna, kungsfågel och gråsiska, eftersom ljuduppspelning använts regelmässigt när dessa fångats. Totalsummor för antal märkta fåglar av respektive art ges i Tabell 1 . Den samlade fångsten av de 40 arterna uppgick till

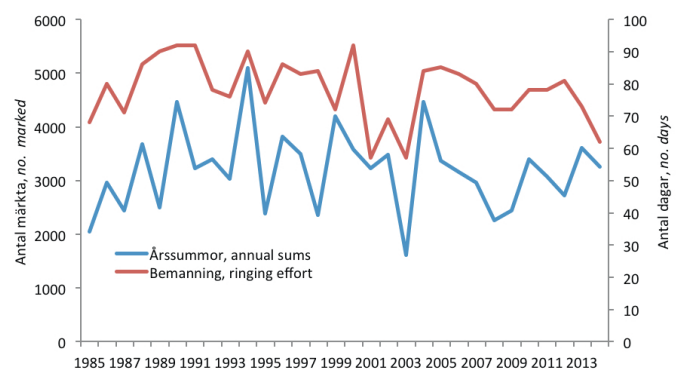

Figur 1. Årssummor av de 40 arterna samt antal bemanningsdagar på Stora Fjäderäggs fågelstation höstarna 1985-2014. Annual ringing sums of the 40 species and number of days with ringing activity at Stora Fjäderägg Bird Observatory, autumns 1985-2014. 
Tabell 1. Märksummor av de 40 mest frekvent fångade fågelarterna på Stora Fjäderäggs fågelstation höstarna 1985-2014 (kungsfågel och gråsiska exkluderade). Autumn ringing totals of the 40 most frequently ringed species at Stora Fjäderägg Bird Observatory 19852014. (Goldcrest and Redpoll excluded).

\begin{tabular}{|c|c|}
\hline Art Species & \\
\hline Större hackspett Dendrocopus major & 2310 \\
\hline Trädpiplärka Anthus trivialis & 476 \\
\hline Ängspiplärka Anthus pratensis & 407 \\
\hline Sädesärla Motacilla alba & 335 \\
\hline Sidensvans Bombycilla garrulus & 917 \\
\hline Järnsparv Prunella modularis & 737 \\
\hline Rödhake Erithacus rubecula & 5097 \\
\hline Blåhake Luscinia svecia & 1038 \\
\hline Rödstjärt Phoenicurus phoenicurus & 1372 \\
\hline Buskskvätta Saxicola rubetra & 85 \\
\hline Stenskvätta Oenanthe oenanthe & 406 \\
\hline Koltrast Turdus merula & 204 \\
\hline Björktrast Turdus pilaris & 1383 \\
\hline Taltrast Turdus philomelos & 998 \\
\hline Rödvingetrast Turdus iliacus & 3437 \\
\hline Ärtsångare Sylvia curruca & 667 \\
\hline Törnsångare Sylvia communis & 246 \\
\hline Trädgårdssångare Sylvia borin & 814 \\
\hline Svarthätta Sylvia atricapilla & 699 \\
\hline $\begin{array}{l}\text { Gransångare Phylloscopus collybita } \\
\text { (abietinus) }\end{array}$ & 837 \\
\hline $\begin{array}{l}\text { Lövsångare Phylloscopus trochilus } \\
\quad \text { (acredula) }\end{array}$ & 32061 \\
\hline Grå flugsnappare Muscicapa striata & 1547 \\
\hline Svartvit flugsnappare Ficedula hypoleuca & 1033 \\
\hline Stjärtmes Aegithalos caudatus & 3415 \\
\hline Svartmes Periparus ater & 1317 \\
\hline Talltita Poecile montanus & 417 \\
\hline Blåmes Cyanistes caeruleus & 4472 \\
\hline Talgoxe Parus major & 4934 \\
\hline Trädkrypare Certhia familiaris & 3574 \\
\hline Törnskata Lanius collurio & 182 \\
\hline Bofink Fringilla coelebs & 836 \\
\hline Bergfink Fringilla montifringilla & 11300 \\
\hline Grönfink Chloris chloris & 298 \\
\hline Grönsiska Carduelis spinus & 1061 \\
\hline Mindre korsnäbb Loxia curvirostra & 282 \\
\hline Rosenfink Carpodacus erythrinus & 198 \\
\hline Tallbit Pinicola enucleator & 424 \\
\hline Domherre Pyrrhula pyrrhula & 3306 \\
\hline Videsparv Emberiza rustica & 1013 \\
\hline Sävsparv Emberiza schoeniclus & 1592 \\
\hline
\end{tabular}

i medeltal 3191 individer per år (variationsbredd 1609-5103) och ingen trend i fångstmängder kan skönjas över de 30 åren ( $p>0.05$, linjär regression).

Årssummorna är signifikant korrelerade med antalet bemanningsdagar $(r=0.44, p<0.02$, Spearman Rank Correlation, Figur 1). Vi har därför valt att beräkna trender för enskilda arter på basis av årsfångstindex, alltså kvoten mellan märksumma och antal bemanningsdagar (fångstinsatsen) det aktuella året. Fångsttrender 1985-2014 för respektive art analyserades med regressionsanalys.

Dessutom gjordes en beräkning av trender för artgrupper genom indelning av de 40 ingående arterna efter flyttningsstrategi. Arterna delades in i grupperna långdistansflyttare, kortdistansflyttare samt invasionsarter/partiella flyttare (Tabell 2). För att minska genomslaget av vanliga arter i beräkningarna av de gruppvisa fångstindexen användes geometriska och inte aritmetiska medelvärden. Som vägledning i gruppindelningen av arterna användes Alerstam (1982), Solonen (1994), Ottvall m.fl. (2009) samt Fransson \& Hall-Karlsson (2008).

\section{Resultat}

Vi fann tio arter med signifikant ökande och åtta arter med signifikant minskande fångsttrender på Stora Fjäderägg 1985-2014 (Tabell 3). Övriga 22 arter visade inte på några signifikanta förändringar i fångstindex.

Talgoxens fångstindex ökade allra mest bland de 40 arterna under 30 -årsperioden (11\%, Tabell 3, Figur 2). Finska häckfågeldata (FHT) visar en tydlig ökning under motsvarande tidsperiod i likhet med SFT 1988-2014 och SFT Norrbotten 1998-2014, men inte SFT 1976-2007. Stjärtmesens fångstindex ökade tydligt 1985-2014 i likhet med SFT 1998-2014. Likaså överensstämmer bofinkens ökning i fångstindex med SFT 1998-2014 och SFT Norrbotten 1998-2014, men inte med SFT 1976-2007. För blåmes, koltrast Turdus merula, svarthätta Sylvia atricapilla (Figur 2) är de positiva trenderna i Fjäderäggsdata, SFT 1976-2007, SFT 1998-2014 och FHT samstämmiga. Ökningen i fångstindex av ärtsångaren Sylvia curruca och sävsparv Emberiza schoeniclus står inte i samklang med SFT eller FHT, tvärtom visar SFT 1998-2014 på en minskad populationstrend hos sävsparven. Rödhakens ökning i fångstindex matchas av samma trend i SFT 1998-2014 och FHT.

Bland arterna utan signifikanta trender i fångstindex 1985-2014 uppvisar gransångaren Phylloscopus collybita (abietinus) och rosenfinken 
Tabell 2. Gruppering av arterna efter flyttningsstrategi.

Grouping of species after migratory strategy.

\begin{tabular}{lll}
\hline Långdistansflyttare & Kortdistansflyttare & Invasionsarter/partiella flyttare \\
Long distance migrants & Short distance migrants & Irruptive species/partial migrants \\
\hline Trädpiplärka Tree Pipit & Ängspiplärka Meadow Pipit & Större hackspett Great Spotted \\
Sädesärla White Wagtail & Järnsparv Dunnock & Woodpecker \\
Blåhake Bluethroat & Rödhake Robin & Sidensvans Waxwing \\
Rödstjärt Redstart & Koltrast Blackbird & Stjärtmes Long-tailed Tit \\
Buskskvätta Whinchat & Björktrast Fieldfare & Svartmes Coal Tit \\
Stenskvätta Wheatear & Taltrast Song Thrush & Talltita Willow Tit \\
Ärtsångare Lesser Whitethroat & Rödvingetrast Redwing & Blåmes Blue Tit \\
Törnsångare Whitethroat & Bofink Chaffinch & Talgoxe Great Tit \\
Trädgårdssångare Garden Warbler & Bergfink Brambling & Trädkrypare Treecreeper \\
Svarthätta Blackcap & Grönfink Greenfinch & Grönsiska Siskin \\
Gransångare Chiffchaff & Sävsparv Reed Bunting & Mindre korsnäbb Crossbill \\
Lövsångare Willow Warbler & & Tallbit Pine Grosbeak \\
Grå flugsnappare Spotted Flycatcher & & Domherre Bullfinch \\
Svartvit flugsnappare Pied Flycatcher & & \\
Törnskata Red-backed Shrike & & \\
Rosenfink Common Rosefinch & & \\
Videsparv Rustic Bunting & & \\
\hline
\end{tabular}

Carpodacus erythrinus intressanta mönster inom perioden. Fångstindex för gransångaren minskade tydligt fram till mitten av 1990-talet och ökade därefter (Figur 2). Gransångaren ökar kraftigt enligt SFT 1998-2014 och SFT Norrbotten 1998-2014. Fångstindex för rosenfink visar på en uppgång fram till 1990, följt av en minskning till början av 2000-talet och därefter åter en ökning (Figur 2). Rosenfinken minskar kraftigt på nationell nivå enligt SFT 1998-2014 och FHT. Större hackspetten Dendrocopus major är en art som fångas i stora antal på Stora Fjäderägg (Tabell 1) med stor mellanårsvariation (Figur 2).

För lövsångare, buskskvätta Saxicola rubetra, videsparv och blåhake Luscinia svecica är fångsttrenderna negativa i likhet med trenderna i SFT och FHT. Fångstindex för videsparven minskade med $4.5 \%$ över hela perioden (Figur 2, Tabell 3). Blåhaken uppvisade den allra största minskningen i fångstindex 1985-2014 (14\%, Tabell 3). Fångstindex för blåhake minskande kraftigt till mitten av 1990-talet för att därefter mer eller mindre plana ut (Figur 2). I likhet med Stora Fjäderägg visar SFT Norrbotten 1998-2014 på minskande trender hos grå flugsnappare Muscicapa striata och svartvit flugsnappare Ficedula hypoleuca. SFT 1998-2014 och FHT visar dock på motsatta trender. Stenskvättans Oenanthe oenanthe minskande fångsttrend på Stora Fjäderägg matchas inte av trenderna i SFT eller FHT. Talltitan Poecile montanus (Figur 2) minskar också enligt FHT och SFT 1976-2007, men uppvisar en starkt ökande trend i SFT Norrbotten 1998-2014.

Andelen arter för vilka fångsttrenderna på Stora Fjäderägg är samstämmiga med trenderna i SFT är högre för dem med ökande fångstindex i SFT 1998-2014 än SFT 1976-2007 (Tabell 4). För arterna med minskande fångsttrender är förhållandet det omvända. För SFT Norrbotten 1998-2014 och FHT är samstämmigheten med Fjäderäggstrenderna bättre för arterna med minskande än ökande index (Tabell 4). Bland förstnämnda är samstämmigheten sämst med SFT 1998-2014. Överlag är samstämmigheten bättre för arter med minskande (69\%) än med ökande (54\%) fångsttrender.

Fångstindex 1985-2014 för kortdistansflyttare minskade med $1,1 \%$ per år medan index för invasionsarter/partiella flyttare ökade med $2,8 \%$ per år. Fångstindex för invasionsarter/partiella flyttare uppvisar ett tydligt oscillerande mönster med ökande amplitud över tiden (Figur 3). För långdistansflyttarna kunde ingen signifikant trend konstateras.

\section{Diskussion}

Vi fann varierande samstämmighet mellan fångsttrenderna på Stora Fjäderägg och svenska och finska häckfågeltaxeringar, med generellt sätt bättre samstämmighet för arter med minskande fångstindex jämfört med sådana med ökande fångstsindex. Diskrepansen mellan SFT 1976-2007 och SFT 
Tabell 3. Trender (årlig förändring i procent) i fångstindex hos 40 fågelarter på Stora Fjäderäggs fågelstation höstarna 1985-2014; nationella semi-kvantitativa trender från punktrutter, standardrutter och andra datakällor 1976-2007 (Ottvall m.fl. 2009), standardrutter i Sverige 1998-2014 (Green \& Lindström 2015a), Norrbotten 1998-2013 (Green \& Lindström 2015b) samt linjeinventeringar i Finland 1986-2012 (Laaksonen \& Lehikoinen 2013). Fetstilade värden är signifikanta på nivån $p<0.05$, kursiverade värden är inte signifikanta. Artlista i fallande ordning efter trenderna på Stora Fjäderägg. N.d. anger att data saknas.

Trends (yearly changes in percent) in capture index for 40 species at Stora Fjäderägg Bird Observatory 19852014; national trends from Swedish (SHT) and Finnish (FHT) breeding bird surveys. Values in bold are significant at the $p<0.05$ level, whereas values in italics are non-significant. $++=$ starkt ökande strongly increasing, $+=$ ökande increasing, $0=$ ingen trend no trend, - = minskande decreasing, $--=$ starkt minskande strongly decreasing. Species ranked after the trends at Stora Fjäderägg. N.d. indicates no data.

\begin{tabular}{|c|c|c|c|c|c|}
\hline $\begin{array}{l}\text { Art } \\
\text { Species }\end{array}$ & $\begin{array}{c}\text { Stora } \\
\text { Fjäderägg } \\
1985- \\
2014\end{array}$ & $\begin{array}{c}\text { Sverige } \\
\text { Sweden } \\
1976- \\
2007\end{array}$ & $\begin{array}{c}\text { Sverige } \\
\text { Sweden } \\
1998- \\
2014\end{array}$ & $\begin{array}{c}\text { Norr- } \\
\text { botten } \\
1998- \\
2013\end{array}$ & $\begin{array}{c}1986- \\
2012\end{array}$ \\
\hline Talgoxe Great Tit & 11 & - & 3.8 & 5.1 & 2.3 \\
\hline Stjärtmes Long-tailed Tit & 8.6 & 0 & 4.1 & N.d. & N.d. \\
\hline Bofink Chaffinch & 7.7 & 0 & 1 & 5.2 & 0 \\
\hline Blåmes Blue Tit & 7 & + & 3.9 & N.d. & 5.7 \\
\hline Grönsiska Siskin & 4.9 & 0 & 0.9 & 2.2 & 0.1 \\
\hline Koltrast Blackbird & 4.8 & + & 1 & N.d. & 4.7 \\
\hline Ärtsångare Lesser Whitethroat & 4.5 & 0 & 0 & N.d. & 0 \\
\hline Sävsparv Reed Bunting & 3.9 & - & -1.5 & -2.1 & -2 \\
\hline Grönfink Greenfinch & 3.2 & ++ & -1.4 & -3 & 5.1 \\
\hline Svarthätta Blackcap & 2.8 & ++ & 6.4 & N.d. & 2.3 \\
\hline Trädkrypare Treecreeper & 2.0 & - & 1.2 & N.d. & 0 \\
\hline Sidensvans Waxwing & 1.8 & 0 & 6.1 & 1.7 & N.d. \\
\hline Järnsparv Dunnock & 1.7 & -- & 2.1 & N.d. & 0 \\
\hline Rödhake Robin & 1.5 & - & 0.7 & N.d. & 1.7 \\
\hline Trädpiplärka Tree Pipit & 1.5 & - & 1.7 & N.d. & -1.7 \\
\hline Gransångare Chiffchaff & 1,4 & -- & 5.3 & 8.9 & 0 \\
\hline Rödstjärt Redstart & 1,1 & -- & 2.3 & N.d. & 1.9 \\
\hline Bergfink Brambling & 0.8 & 0 & -1.8 & -2.2 & -0.2 \\
\hline Trädgårdssångare Garden Warbler & 0.8 & 0 & 1.6 & N.d. & 0.3 \\
\hline Taltrast Song Thrush & 0.7 & 0 & 2.5 & 2.9 & 0.1 \\
\hline Domherre Bullfinch & 0.3 & - & 1.2 & -4.2 & -0.8 \\
\hline Törnsångare Whitethroat & 0,3 & 0 & 1.2 & N.d. & 0 \\
\hline Större hackspett Gr. Sp. Woodpecker & -0.3 & - & 3.5 & 6.9 & 2.1 \\
\hline Mindre korsnäbb Crossbill & -0.4 & 0 & 6.6 & 15 & -0.2 \\
\hline Rosenfink Common Rosefinch & -0.4 & ++ & -4.8 & N.d. & -2.9 \\
\hline Sädesärla White Wagtail & -1.1 & - & 0.1 & N.d. & N.d. \\
\hline Björktrast Fieldfare & -1.7 & + & -2.5 & -2.4 & 1.4 \\
\hline Lövsångare Willow Warbler & -1.7 & 0 & -1.5 & -4.8 & -1.6 \\
\hline Tallbit Pine Grosbeak & -1.7 & & -5.7 & N.d. & N.d. \\
\hline Ängspiplärka Meadow Pipit & -1.8 & 0 & -1.3 & -4.1 & -1.3 \\
\hline Svartmes Coal Tit & -2.3 & - & 0.2 & N.d. & -1.2 \\
\hline Rödvingetrast Redwing & -3.0 & 0 & -0.6 & -1.6 & 0 \\
\hline Grå flugsnappare Spotted Flycatcher & -3.4 & 0 & 2.5 & -3.8 & 0.7 \\
\hline Törnskata Red-backed Shrike & -3.4 & - & -0.3 & N.d. & 0.4 \\
\hline Videsparv Rustic Bunting & -4.5 & - & -4.8 & -3.1 & -6.2 \\
\hline Svartvit flugsnappare Pied Flycatcher & -4.9 & - & 1 & -2.7 & 0.8 \\
\hline Buskskvätta Whinchat & -5.9 & -- & -1.2 & N.d. & -2 \\
\hline Stenskvätta Wheatear & -12 & - & -0.6 & N.d. & N.d. \\
\hline Talltita Willow Tit & -13 & -- & 0.7 & 4 & -1.5 \\
\hline Blåhake Bluethroat & -14 & - & -1.1 & N.d. & -3.1 \\
\hline
\end{tabular}



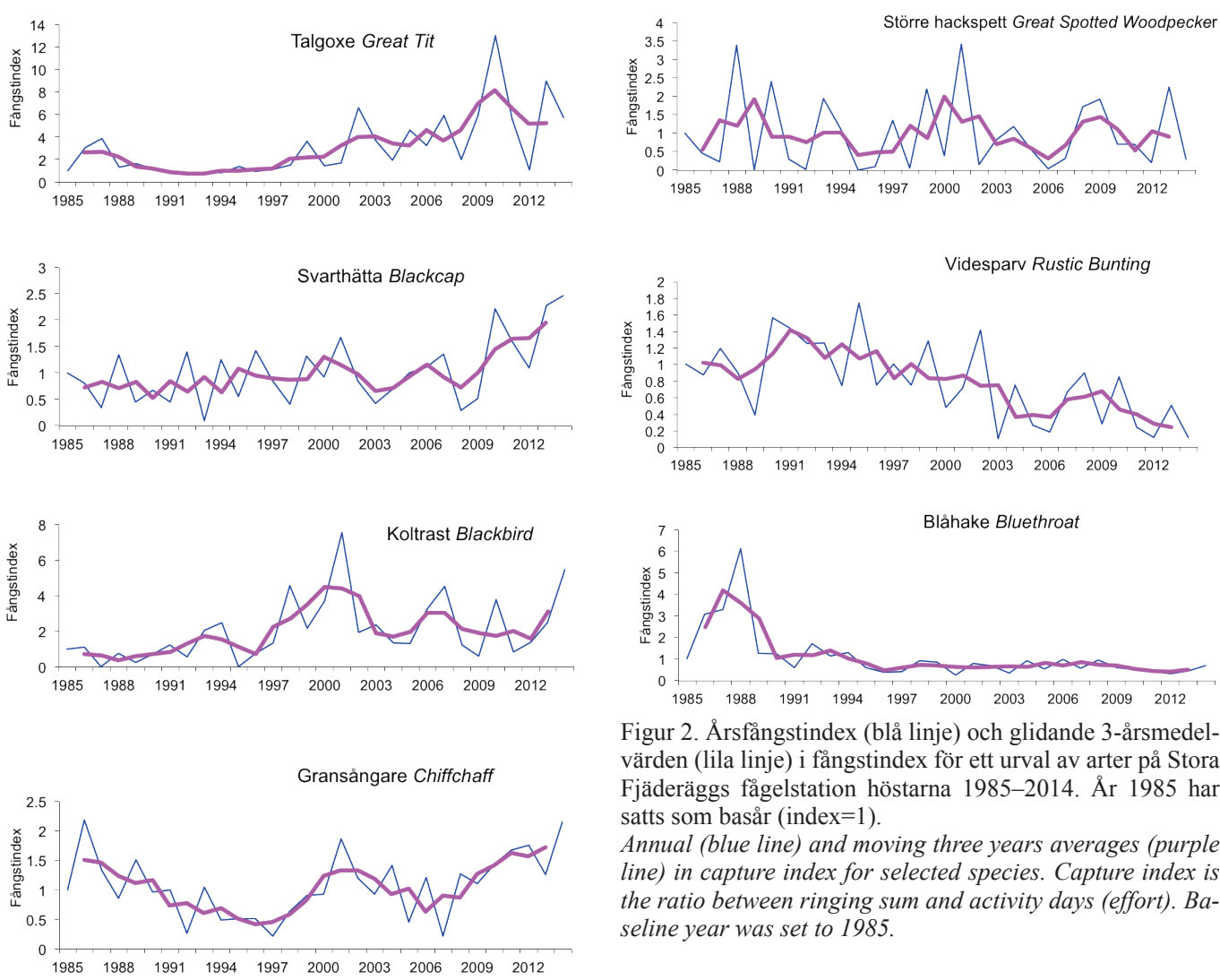

Figur 2. Årsfångstindex (blå linje) och glidande 3-årsmedelvärden (lila linje) i fångstindex för ett urval av arter på Stora Fjäderäggs fågelstation höstarna 1985-2014. År 1985 har satts som basår (index $=1$ ).

Annual (blue line) and moving three years averages (purple line) in capture index for selected species. Capture index is the ratio between ringing sum and activity days (effort). Baseline year was set to 1985 .
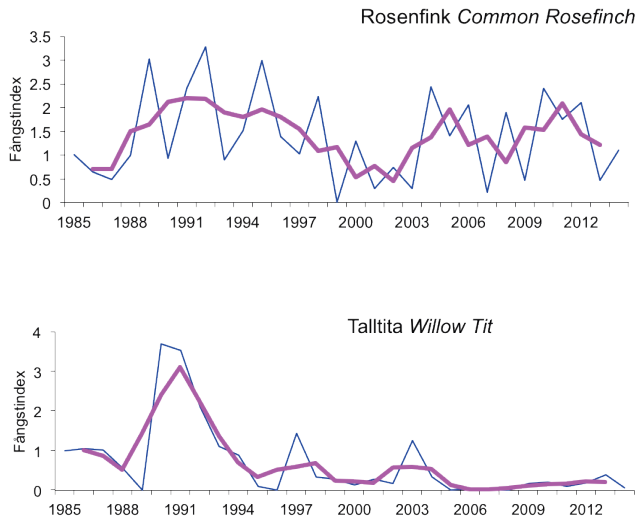

sen mellan fångsttrenderna för ökande arter på Stora Fjäderägg och SFT 1976-2007. Å andra sidan är överensstämmelsen mellan fångsttrenderna för minskande arter och SFT bättre för perioden 1976-2007 än 1998-2014. SFT 1998-2014 avviker i detta avseende även från SFT Norrbotten 1998-2014 och FHT. SFT Norrbotten 1998-2014 visar i likhet med Stora Fjäderäggsdata, men till skillnad från SFT 1998-2014, negativa trender för grå flugsnappare och svartvit flugsnappare. Även tidigare studier har visat på motstridiga trender mellan ringmärkningsdata och häckfågeldata i fråga om svartvit flugsnappare (Karlsson m.fl. 2002b). Möjligen indikerar det att ringmärkningsdata är dåliga indikatorer för populationsförändringar hos flugsnapparna. Det kan även indikera att det finns inomartsskillnader i trender mellan norra och södra Sverige som inte syns i de nationella redovisningarna. Den generellt goda samstämmigheten med trenderna i FHT kan hänga samman med att Stora Fjäderägg i väsentlig grad berörs av flyttfågelströmmar med ett östligt ursprung under hösten.

Den exceptionella ökningen i fångstindex av talgoxe på Stora Fjäderägg 1985-2014 matchas

1998-2014 kan ha flera orsaker, bl.a. kan de jämförelsevis få rutterna i norra Sverige under den första delen av 30-årsperioden inte ha fått något starkt genomslag i de nationella trendberäkningarna. Det skulle kunna förklara den dåliga överensstämmel- 
Tabell 4. Kvalitativ jämförelse av fångsttrender hos signifikant ökande (+) respektive minskande (-) arter på Stora Fjäderägg 1985-2014 och SFT 1976-2007 (Ottvall m.fl. 2009), SFT 1998-2014 (Green \& Lindström 2015a), Norrbotten 1998-2014 (Green \& Lindström 2015b) samt FHT 1986-2012 (Laaksonen \& Lehikoinen 2013). N.d. betyder inga data.

Qualitative comparison of trends in capture indices at Stora Fjäderägg and trends from breeding bird surveys in Sweden and Finland. N.d. means no data.

\begin{tabular}{lccccc}
\hline & Stora & SFT & SFT & Norrbotten & FHT \\
& Fjäderägg & $1976-$ & $1998-$ & $1998-$ & \\
& $1985-2014$ & 2007 & 2014 & 2014 & \\
\hline Talgoxe Great Tit & + & - & + & + & + \\
Stjärtmes Long-tailed Tit & + & 0 & + & N.d. & N.d. \\
Bofink Chaffinch & + & 0 & + & + & 0 \\
Blåmes Blue Tit & + & + & + & N.d. & + \\
Grönsiska Siskin & + & 0 & + & + & 0 \\
Koltrast Blackbird & + & + & + & N.d. & + \\
Ärtsångare Lesser Whitethroat & + & 0 & 0 & 0 & - \\
Sävsparv Reed Bunting & + & - & - & - & - \\
Svarthätta Blackcap & + & + & + & N.d. & + \\
Rödhake Robin & + & - & + & N.d. & + \\
Lövsångare Willow Warbler & - & 0 & - & - & - \\
Grå flugsnappare Spotted Flycatcher & - & 0 & + & - & 0 \\
Videsparv Rustic Bunting & - & - & - & - & - \\
Svartvit flugsnappare Pied Flycatcher & - & - & + & - & 0 \\
Buskskvätta Whinchat & - & - & - & N.d. & - \\
Stenskvätta Wheatear & - & - & 0 & N.d. & N.d. \\
Talltita Willow Tit & - & - & 0 & + & - \\
Blåhake Bluethroat & - & - & - & N.d. & - \\
\hline Kvot ökande Proportion increasing & $10 / 10$ & $3 / 10$ & $8 / 10$ & $3 / 5$ & $5 / 9$ \\
Kvot minskande Proportion decreasing & $8 / 8$ & $6 / 8$ & $4 / 8$ & $4 / 5$ & $5 / 7$ \\
\hline
\end{tabular}

bättre av trenden i SFT Norrbotten 1998-2014 än i SFT 1998-2014. Det kan hänga samman med skillnader i trender mellan norra och södra Sverige. Till exempel har varmare (mer sydliga) arter spridit sig norrut och ökat i norr (Green \& Lindström 2015b). Talgoxe kan vara en sådan art där en ökning i norr kanske ännu inte slagit igenom ordentligt i rikstrenden. Stjärtmesen ökar även på standardrutterna i SFT 1998-2014. Av just stjärtmes har vi flera intressanta kontroller på Stora Fjäderägg av fåglar märkta tidigare samma höst längre söderut längs Finlands kust, i Estland och i ryska Karelen. Det kan skilja dagar och i extremfall endast timmar mellan märkning och kontroll vilket ger en bild av en koncentrerad snabb nordlig-nordvästlig flyttrörelse med ursprung utanför Sveriges gränser. Samma mönster går för övrigt igen hos blåmesen och talgoxen. Svarthättan har en mycket positiv utveckling i Sverige, inte minst i norra Sverige (Green \& Lindström 2015a). Rosenfinken uppvisar ingen trend i Stora Fjäderäggsdata 1985-2014 vilket står i kontrast med den stadiga minskningen av rosenfink i SFT 1998-2014 och FHT. I Stora
Fjäderäggs fall tycks det vara så att perioderna med ökning och minskning tagit ut varandra. Analysperiodens startpunkt och längd har stor betydelse för skattningen av trender, och kan i gransångarens fall förklara varför den tydliga ökningen i index i SFT 1998-2004 inte syns i Fjäderäggsdata som startade tidigare och som sträcker sig över en mycket längre tidsperiod. Den negativa fångsttrenden för videsparven överensstämmer väl med den negativa

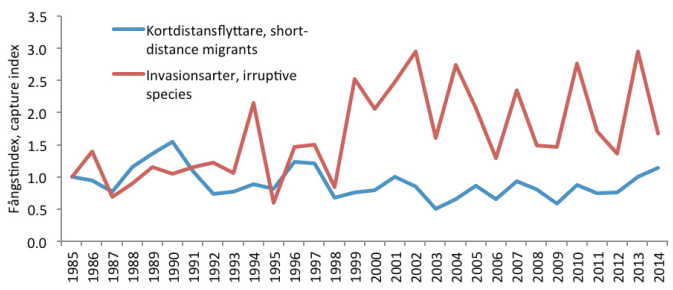

Figur 3. Årsfångstindex för arterna grupperade efter flyttningsstrategi med 1985 som basår.

Capture index for species grouped after migratory strategy with 1985 as baseline year. 
populationsutvecklingen generellt i Fennoskandien (Dale \& Hansen 2013). En snabb initial minskning i fångstindex var tillräckligt kraftig för att generera en stark negativ trend för hela 30-årsperioden hos blåhaken. Det finns inga storskaliga häckfågeltaxeringar från 1980-talet att jämföra med, men häckfågeldata från Ammarnäsområdet 1963-1999 visar inte på någon långsiktig trend i populationstäthet hos blåhake i detta område (Enemar m.fl. 2004). Inte heller standardiserade fångstdata från Ottenby fågelstation visar på någon långsiktig trend perioden 1979-2008 (Svanberg \& Waldenström 2011). Fjällbjörkmätaren Epirrita autumnata, en viktig födokälla för insektsätande fåglar i fjällnära miljö, hade en markerad topp i mitten av 1980-talet i Ammarnäsområdet, vilken tidsmässigt sammanföll med toppen för fångstindex hos blåhake på Stora Fjäderägg 1985-2014 (Figur 2). Man kan därför spekulera i om en hög ungfågelproduktion bidrog till de höga märksummorna i mitten av 1980-talet. Senare utbrott av fjällbjörkmätare på 1990-talet har varit mycket mindre omfattande (Enemar m.fl. 2004).

Den signifikanta minskningen i fångstindex hos gruppen kortdistansflyttare 1985-2014 förtjänar att nämnas. De tre arterna i gruppen med signifikanta trender var alla ökande (rödhake, koltrast och sävsparv), men sammantaget och med hänsyn taget till skillnader i märksummor var trenden alltså negativ för gruppen som helhet. Det bör dock påpekas att trenden är svag, 1,1\% per år. Jämförelser med SFT går inte att göra rakt av eftersom gruppindelningarna ser olika ut, men i SFT 1998-2014 ökar fem av arterna medan sex minskar, och i SFT Norrbotten 1998-2014 är motsvarande siffror en respektive tre (av sju arter för vilka data redovisas). Här tycks alltså trenderna mer eller mindre ta ut varandra. Till skillnad från andra studier fann vi ingen minskning i fångsttrend hos långdistansflyttarna som grupp betraktat. Bland arter med signifikanta trender fann vi två med ökande och sju med minskande trender. Resterande åtta arter uppvisade ingen signifikant trend. Bland arterna som övervintar söder om Sahara, så kallade Afro-Palearktiska arter, uppvisade dock fem av åtta av arter i gruppen signifikant negativa trender, alltså vad som kunde förväntas för denna delgrupp baserat på andra studier (Vickery m.fl. 2014). Fångsttrenderna för arter som flyttar mot sydost och ost, i Stora Fjäderäggs fall blåhake, rosenfink och videsparv, var negativa i två fall av tre vilket förtjänar att nämnas. Fångsttrenden för invasionsarter/partiella flyttare ökade under 30-årsperioden. Denna grupp utgjorde $28 \%$ av den totala fångstsumman av de 40 arterna 1985-2014, en an- senlig andel med andra ord. Fångstindex i gruppen uppvisade en påtaglig dynamik med över tiden allt mer markerade toppar. Mesarnas andel av gruppen var 36\% och här ingår stjärtmesen, blåmesen och talgoxen där återfynd antyder att en stor del rekryteras utanför landet. I denna grupp är det därför tänkbart att fångstindex speglar mellanårsvariationen i reproduktion hos ökande populationer på en större geografisk skala eller ett förändrat flyttningsbeteende. Vädret kan också spela in då ett större inflöde av mesar noteras under höstar med ihållande sydliga vindar. Talltitan avviker från mönstret för gruppen som helhet genom ett minskande fångstindex och en över tiden alltmer avtagande mellanårsvariation (Figur 2). Minskningen är dock helt i linje med den minskande långsiktiga trend som uppvisas av talltitan i såväl SFT 1976-2007 som FHT men inte SFT Norrbotten 1998-2014.

\section{Felkällor}

Tolkning av populationstrender på basis av ringmärkningsdata måste göras med försiktighet. Nedan diskuterar vi ett antal potentiella felkällor och hur de kan ha påverkat resultaten och vår tolkning av dessa. I strikt mening var höstfångsten på Stora Fjäderägg 1985-2014 inte standardiserad. Variation i fångstinsats kan få stort genomslag i korta dataserier, men med långa tidsserier jämnas detta ut och blir mindre betydelsefullt. Eftersom det inte skett någon systematisk förändring $\mathrm{i}$ fångstrutinerna eller bemanning över tiden betraktar vi variation av detta slag som ett slumpmässigt fel som i likhet med väder skapar brus och minskar precisionen i data. Vi har dessutom korrigerat för antalet bemanningsdagar genom att ta med det $\mathrm{i}$ fångstindex. Förändringar i miljön kan också ha påverkat fångstsiffrorna. Igenväxningen av öns tidigare till största delen öppna hedmarker har varit påtaglig under 30-årsperioden (även om senare års röjningsinsatser har sökt motverka detta). En minskad fångstbarhet kopplat till igenväxning kan ha bidragit till den kraftiga minskningen i fångstindex för stenskvätta, en art bunden till öppen mark. En annan påtaglig förändring i miljön är den accelererande igenväxningen av öns stränder med gråal $\mathrm{Al}$ nus incana och viden Salix spp. som ett resultat av upphörd beteshävd och vedfångst samt den pågående eutrofieringen av Bottniska viken. Detta tror vi är en sannolik förklaring till ökningen i fångstindex för sävsparv som står i kontrast till trenderna $\mathrm{i}$ SFT och FHT. Intressant att notera i sammanhanget är att inte blåhaken och videsparven, som föredrar att rasta i samma miljö under hösten, verkar ha på- 
verkats i samma riktning. Ökad förbuskning och beskogning bedöms i övrigt inte ha inverkat på de trender som är tydliga. Ett antal i huvudsak skogslevande tättingar finns häckande i Stora Fjäderäggs barrskogsdungar men öns populationer är små och de utgör sannolikt bara en marginell andel av antalet fångade individer.

Ringmärkningsdata $\mathrm{i}$ form av långa tidsserier kan ge värdefulla bidrag till populationsstudier genom att fylla ut dataluckor och som komplement till häckfågelstudier. Ringmärkningsdata kan också signalera vad som sker med fågelpopulationerna på större geografisk skala, i synnerhet om återfynd och kontroller samtidigt möjliggör en geografisk koppling till de ringmärkta fåglarna. Även om ringmärkningsdata inte okritiskt bör användas för att tolka populationsförändringar så är det påfallande hur överensstämmande många av trenderna i fångstindex på Stora Fjäderägg och trenderna i häckfågeltaxeringarna i Sverige och Finland är, särskilt bland arterna med minskande trender. Det finns därför goda skäl att tro att fångsttrenderna på Stora Fjäderägg speglar verkliga förändringar i populationsstorlek. Även utan strikt standardiserad fångst har fågelstationer med långsiktig verksamhet goda möjligheter att bidra med värdefulla data till miljöövervakningen. Detta är inte minst viktigt i mer ornitologglesa regioner där antalet fågelstationer och märkplatser är få och utspridda.

\section{Tack}

Vårt tack går till alla som frivilligt ställt upp med tid och kunskap i ringmärkningen på Stora Fjäderäggs fågelstation. Ingen nämnd och ingen glömd, men vi vill rikta ett särskilt tack till Björn Olsen för insatserna i samband med fågelstationens tillkomst. Vi är tacksamma för kommentarer på manus från Richard Ottvall.

\section{Referenser}

Alerstam, T. 1982. Fågelfyttning. Signum. Lund.

Berthold, P. \& Fiedler, W. 2005. Changes in the populations of small birds in central Europe as evidenced by 32 years trapping data: numbers are mostly declining. Vogelwarte 43: 97-102.

Busse, P. 1990. Studies of long-term population dynamics based on ringing data. The Ring 13: 221-234.

Dale, S. \& Hansen, K. 2013. Population decline in the Rustic Bunting Emberiza rustica in Norway. Ornis Fennica 90: 193-202.

Dunn, E. H., Hussel, D. J. T. \& Adams, R. J. 1997. Monitoring songbird population change with autumn mist netting. Journal of Wildlife Management 61: 389-396.

Elmberg, J., Lindberg, N. \& Edenius, L. 2005. Vart tog de vägen? En översikt av återfynd av fåglar ringmärkta på Stora Fjäderägg. Fåglar $i$ Västerbotten 30(4): 4-9.

Enemar, A., Sjöstrand, B., Andersson, G. \& von Proschwitz, T. 2004. The 37-year dynamics of a subalpine passerine bird community, with special emphasis on the influence of environmental temperature and Epirrita autumnata cycles. Ornis Svecica 14: 63-106.

Fransson, T. \& Hall-Karlsson, S. 2008. Svensk ringmärkningsatlas. Vol. 3. Stockholm.

Green, M. \& Lindström, Å. 2015a. Övervakning av fåglarnas populationsutveckling. Arsrapport för 2014. Rapport, Biologiska institutionen, Lunds universitet. $86 \mathrm{~s}$.

Green, M. \& Lindström, Å. 2015b. Häckande fåglar $i$ Norrbottens län - Trender för arter och miljöindikatorer baserade på standardrutter 1998-2013 och punktrutter i odlingslandskapet 2007-2013. Länsstyrelsens rapportserie no. 2, 2015. Rapport, Länsstyrelsen i Norrbottens län. 23 s. (exkl. bilagor).

Hochachka, W. M. \& Fielder, W. 2008. Trends in trappability and stop-over duration can confound interpretations of population trajectories from long term migration ringing sites. Journal of Ornithology 149: 375-391.

Jonzén, N., Lindén, A., Ergon, T., Knudsen, E., Vik, J. O., Rubolini, D., Piacentini, D., Brinch, C., Spina, F., Karlsson, L., Stervander, M., Andersson, A., Waldenström, J., Lehikoinen, A., Edvardsen E., Solvang, R. \& Stenseth, N. C. 2006. Rapid advance of spring arrival dates in longdistance migratory birds. Science 312: 1959-1961.

Karlsson, L., Ehnbom, S., Persson, K. \& Wallinder, G. 2002a. Changes in numbers of migrating birds at Falsterbo, during 1980-1999, as reflected by ringing totals. Ornis Svecica 12: 113-137.

Karlsson, L., Ehnbom, S. \& Wallinder, G. 2002b. A comparison between ringing totals at Falsterbo, SW Sweden, ringing totals at Ottenby, SE Sweden, and point counts from the Swedish Breeding Bird Census during 20 years (1980-1999). Ornis Svecica 15: 183-205.

Laaksonen, T. \& Lehikoinen, A. 2013. Population trends in boreal birds: continuing declines in agricultural, northern, and long-distance migrant species. Biological Conservation 168: 99-107.

Ottvall, R., Edenius, L., Elmberg, J., Engström, H., Green, M., Holmqvist, N., Lindström, Å., Pärt, T. \& Tjernberg, M. 2009. Population trends of the birds. Ornis Svecica 19: 117-192.

Solonen, T. 1994. Structure and dynamics of the Finnish avifauna. Memoranda Soc. Fauna Flora Fennici 24: 281-294.

Stervander, M., Lindström, Å., Jonzén, N. \& Andersson, A. 2005. Timing of spring migration in birds: long-term trends, North Atlantic Oscillation and the significance of different migration routes. Journal of Avian Biology 36: 210-221.

Svanberg, S. \& Waldenström, J. 2011. Population fluctuations and timing of spring migration of the Scandinavian Bluethroat Luscinia svecica svecica at Ottenby Bird Observatory, Sweden, 1955-2008. Ornis Svecica 21: 92-100.

Svensson, S. \& Andersson, T. 2013. Population trends of birds in alpine habitats at Ammarnäs in southern Swedish Lapland 1972-2011. Ornis Svecica 23: 81-107.

Vickery $\mathrm{m}$ fl. 2014. The decline of Afro-Palearctic migrants and an assessment of potential causes. Ibis 156: 1-22. 


\section{Summary}

Long data series that describe the development of bird populations over time are important to understand the internal dynamics in bird communities, identify ecological patterns and processes, knowledge gaps and potential threats. Breeding bird surveys provide the most reliable data for population trend analysis, but surveys of this kind have a relatively short history in northern Sweden. Trend analysis from northern Sweden is particularly important as bird populations here seem to develop more negatively compared to southern Sweden. We also know less about northern bird populations in general than in other parts of Europe. Ringing data have been used for analysis of population change and to increase our understanding of important processes in bird populations. Trend analysis based on ringing data covering 20 years has been presented from Ottenby and Falsterbo Bird Observatories in southern Sweden, but similar studies have not been published from bird observatories in northern Sweden. Recovery data of birds ringed at Stora Fjäderägg Bird Observatory, NE Sweden, indicates little exchange of birds with southern Sweden, thus warranting the need for regionalized analyses. We present trends in autumn ringing data based on 30year data series for 40 more frequently occurring species at Stora Fjäderägg Bird Observatory, NE Sweden, 1985-2014. These trends are compared with population trends for breeding bird surveys from Sweden and Finland for the same time period.

\section{Material and methods}

Stora Fjäderägg is a c. $1 \times 1 \mathrm{~km}$ large, mostly barren island, located in the Gulf of Bothnia between Sweden and Finland $\left(63^{\circ} 49^{\prime} \mathrm{N}, 21^{\circ} 00^{\circ} \mathrm{E}\right)$. Stora Fjäderägg was established as a bird observatory in 1984 , but more regular ringing activity started one year later. Twenty to 25 mist nets were used from dawn till around lunch time. The observatory is managed by volunteers, which results in some variation in ringing activity between years; the mean number of days with ringing activity in August-October 1985-2014 was 78, with no trend over time. Ringing totals for each of the 40 species are given in Table 1. Annual ringing totals averaged 3191 birds (range 1609-5103), with no trend over time. The annual ringing totals were correlated with number of days with ringing activity. We therefore employed a yearly capture index in estimating trends, taken as the ratio between number of birds ringed and days with ringing activity.
Significance $(p<0.05)$ of a linear trend being separated from null over the 30 years was assessed by means of regression analysis.

\section{Results}

Ten species showed an increase and eight species a decrease in capture index at Stora Fjäderägg 19852014 (Table 2). The remaining 22 species had no significant trend in capture index. Great Tit showed the strongest increase in capture index throughout the 30-year period (Table 2, Fig. 2), followed by Long-tailed Tit and Chaffinch. The positive trend in capture index for Blue Tit and Blackcap coincided with similar trends in Swedish and Finnish long-term and short-term breeding bird surveys. Among species with no significant trends, Chiffchaff and Common Rosefinch showed interesting patterns within the 30-year period. Capture index for the Chiffchaff decreased 1985-1995, and thereafter rose (Figure 2). Capture index for Common Rosefinch showed an oscillating pattern with an early increase, followed by a decrease and a second increase. Capture indices for Whinchat, Rustic Bunting, Willow Tit and Bluethroat were negative, similar to the trends observed in the Swedish and Finnish long-term breeding bird survey data. The Bluethroat showed a rapid dramatic initial decrease phase in capture index that quickly leveled off (Figure 2). A 1.1\% annual decrease was seen in capture index for short-distance migrants and a $2.8 \%$ annual increase in irruptive species/partial migrants (Figure 3 ). No significant trend in capture index was seen in long-distance migrants.

\section{Discussion}

In general, there was a better match between capture indices at Stora Fjäderägg and long- and shortterm Swedish and Finnish breeding bird survey data for species declining than for those increasing. One explanation for a poor match between the capture indices and long-term Swedish breeding survey data might be that the number of standardized routes in the Swedish breeding bird surveys is relatively few from northern Sweden before year 2000 and therefore contributes little to the long-term national trend estimates. A high correspondence with the Finnish breeding survey data could be related to a high influx of birds with an eastern origin at Stora Fjäderägg during autumn.

Long-tailed Tit is a scarce breeding bird in northern Sweden, which indicates that most of the trapped individuals have a more distant origin. The 
decrease in capture index for the Rustic Bunting echoes the strong negative population trend reported from Fennoscandia. The Bluethroat showed the strongest decrease in capture index of all analyzed species. Interestingly, this was due to a very strong initial decrease that quickly leveled off. This could possibly be related to a pronounced peak in production of Epirrita autumnata caterpillars at the breeding grounds in the mid-1980s which coincided with the peak in numbers of Bluethroats ringed at Stora Fjäderägg. The decrease in capture index for short-distance migrants was puzzling, but it is difficult compare trends with other studies as group delineations vary. Likewise, the non-significant trend in long-distance migrants was puzzling. However, among the Afro-Palearctic migrant subgroup consisting of eight species, six displayed significant negative trends in capture index. Among species with an eastern and/or south-eastern migratory route, two out of three had negative capture indices. Capture index for irruptive species/partial migrants increased markedly over the 30-year period. This group constituted a substantial part, $28 \%$, of the ringing totals 1985-2014. Capture index for this group showed pronounced yearly fluctuations with a tendency for increasing amplitude over time. For this group one might hypothesize that capture index reflects between-year variation in reproduction within increasing populations over larger spatial scales or altered migration patterns. Willow Tit deviates from the pattern in the group with decreasing and progressively diminishing amplitude in capture index. However, this is in line with the overall long-term population trends for Willow Tit in Sweden and Finland.

\section{Error sources}

Interpretation of population changes based on ringing data must be done with caution. The ringing activity at Stora Fjäderägg Bird Observatory was not formally standardized during the 30-year period; by and large this is attributable to the fact that the ringing activity was based on voluntary work. Variation in capture effort can have a large impact on short data series, but over time such discrepancies will be become progressively less important. Since there has been no change in ringing effort over the years, we regard this source of variation, like weather, as a random error factor increasing the noise and lower the precision in the data. In addition, we corrected the data for variation in capture effort by incorporating number of days with ringing activity in the capture indices. Changes in habitat 1985-2014 may have also affected the results. Shrub encroachment of earlier open heath land probably reduced suitability of Stora Fjäderägg as a stop-over site for open-ground species. This may be part of the explanation for the strong decrease in capture index of Northern Wheatear. For species preferring open habitat it cannot be ruled out that the reduction in capture index reflects changes in trappability. A similar source of error is the ongoing encroachment of shorelines with Grey alder and willows. We believe that this could explain the increase in capture index of Reed Bunting, which stands in strong contrast with the trends in the national breeding survey data. Interestingly Bluethroat and Rustic Bunting, which prefers the same kind of habitat during migration, did not show the same pattern in capture index. Most species of forest habitats only have small populations on the island and they probably make up a marginal part of the number of individuals trapped. 